\title{
LA HISTORIA EN LA VENTANA: CONFIGURACIÓN Y REPRESENTACIÓN DEL TIEMPO EN LA VENTANA
} ALBERTIANA

The history on the window: configuration and representation of time in the albertian window

\author{
Macarena García Moggia*
}

Resumen

Este artículo ofrece una lectura de la pintura renacentista según los principios establecidos por Leon Battista Alberti, uno de los primeros teóricos de la perspectiva en cuyo Tratado de pintura (1435) se refiere al cuadro como "una ventana abierta a la historia". El concepto de historia empleado por Alberti, que se presta a numerosas interpretaciones, es abordado a partir de las reflexiones de Erwin Panofsky en torno a la perspectiva como "forma simbólica", avanzando hacia una hipótesis en torno al carácter simbólico de la configuración y representación del tiempo en el cuadro-ventana albertiano.

Palabras clave: Cuadro, ventana, espacio, tiempo, historia.

Abstract

This paper offers a reading of the renaissance painting according to the principles established by Leon Battista Alberti, one of the first theorists of perspective in painting which Trattato della pittura (1435) define the frame of the painting as "an open window into history". The concept of history employed by Alberti, which lends itself to many interpretations, is approached from the reflections of Erwin Panofsky about the prospect as "symbolic form", moving towards a hypothesis about the symbolic nature of the configuration and representation of time in the alberti's window.

Key words: Painting, frame, window, space, time, history.

\section{I.}

En su famoso tratado De Pictura, de 1435, Leon Battista Alberti habla del cuadro como de "una ventana abierta a la istoria", donde la istoria quizás no sea exactamente el relato, la anécdota o el tema del cuadro sino más bien, como ha señalado Jean-Louis Schefer, traductor del tratado de Alberti al francés, "el objeto mismo de la pintura", vale decir, "lo que puede ser objeto de una narración o de una descripción" (Schefer, 115) ${ }^{1}$.

Esta arista del texto de Alberti ha sido, en general, menos comentada; suele privilegiarse la dimensión espacial de la intuición perspectiva por sobre la dimensión temporal que involucra. Erwin Panofsky, sin ir más lejos, da inicio a su texto señero

\footnotetext{
${ }^{1}$ La traducción de este y los siguientes pasajes citados cuya fuente está en francés o inglés, es nuestra.
} 


\section{Macarena García Moggia}

acerca del problema haciendo referencia a la concepción albertiana del cuadro como una ventana "a través de la cual nos parezca estar viendo el espacio" (11), espacio que es negado en su condición material para transformarse en un "plano figurativo", sobre este se proyecta un espacio unitario y homogéneo que nunca es dado sino un construido como un Quantum continumm que omite, por ejemplo, "que vemos con dos ojos en constante movimiento y no con uno fijo" (Panofsky, 14-15). Dicha concepción del espacio conlleva, según Panofsky, una determinada concepción de la relación entre el sujeto y el mundo exterior, la que presenta un doble filo:

(...) por un lado reduce los fenómenos artísticos a reglas matemáticas sólidas y exactas, por otro las hace dependientes del hombre, del individuo, en la medida en que las reglas se fundamentan en las condiciones psicofisiológicas de la impresión visual y en la medida en que todo su modo de actuar está determinado por la posición de un "punto de vista" subjetivo elegido a voluntad (Panofsky, 49).

Consolidación y sistematización del mundo externo, expansión de la esfera del yo: entre ambos polos, la ventana perspectiva aparece como un dispositivo que modela tanto al espacio proyectado como al observador mismo, cuya experiencia de la visión pasa por reglas matemáticas que facultan una representación del espacio que deviene, en adelante, simbólica. De ahí el título que Panofsky escoge para su estudio: $\mathrm{La}$ perspectiva como forma simbólica:

Si la perspectiva no es un momento artístico -dice Panofsky-, constituye, sin embargo, un momento estilístico y, utilizando el feliz término acuñado por Ernst Cassirer, debe servir a la historia del arte como una de aquellas "formas simbólicas" mediante las cuales "un particular contenido espiritual se une a un signo sensible concreto y se identifica íntimamente con él" (24).

$\mathrm{Al}$ acuñar el término "forma simbólica", Panofsky realiza un desplazamiento de un concepto ampliamente desarrollado por Ernst Cassirer en su Filosofia de las Formas Simbólicas, cuyo primer volumen sale a la luz en 1923, cuatro años antes de la primera publicación del ensayo de Panofsky, en 1927. El estudio realizado por Cassirer propone, a su vez, una reapropiación de los resultados esenciales del trabajo crítico llevado a cabo por Kant en el campo del conocimiento, trasladándolos al problema general de la cultura. Si la Crítica de la razón pura había brindado los fundamentos teóricos para una ciencia que renuncia a la pretensión de captar la realidad objetiva de manera "inmediata", de lo que se sigue que toda objetivación del conocimiento nunca sería más que una "mediación", es decir, un acto de la mente cognoscente, no por ello esa ciencia renuncia a "la exigencia fundamental de unidad" que el conocimiento conlleva, dirá Cassirer, agregando que esa unidad que no puede encontrarse ante nuestros ojos se encuentra en nuestros ojos, vale decir, en la mente que conoce, cuyo límite es el "principio de razón suficiente", que es "la meta esencial del conocimiento: unir lo particular a una ley y un orden que tengan la forma de la universalidad" (18). Así, no puede trazarse, según Cassirer, una “tajante línea 
divisoria entre el campo de lo sensible y el campo de lo inteligible, en forma tal que ambos quedaran como esferas aisladas entre sí, correspondiendo a cada una de ellas una especie propia e independiente de realidad" (156): la crítica del conocimiento kantiana ha mostrado que la mera sensación de ningún modo es resultado de la experiencia inmediata sino el producto de una abstracción o proceso de síntesis que reposa sobre las formas puras de la sensibilidad - espacio y tiempo- $\mathrm{y}$ funda el acto del conocimiento.

La noción de "Forma Simbólica" empleada por Cassirer, y luego por Panofsky, seguiría la lógica de este esquema trascendental. Según ha señalado George Didi-huberman en un acucioso análisis acerca de la evolución e implicancias para la historia del arte del concepto en cuestión:

Como las Ideas kantianas, en efecto, las formas simbólicas de Cassirer y de Panofsky habrán sido aprehendidas en la óptica de principios reguladores que "sistematizan una síntesis"; al igual que las Ideas, primero habrán sido pensadas desde el punto de vista de la subjetividad - como actos del mundo de la cultura y no del mundo a secas-, pero luego re-objetivadas, si podemos decirlo, en su autoridad de reglas y en su vocación en la "unidad final" de las cosas (174).

Sin embargo, que el arte en general, tanto como el lenguaje y el mito operen, según Cassirer, de acuerdo con formas simbólicas, reintroduce un "tercer término" en el esquema trascendental kantiano, a saber, la cultura. Jean-Louis Déotte se ha referido a este aspecto con detenimiento. En su estudio de lo que él llama "aparatos", que en pocas palabras pueden definirse como dispositivos culturales capaces de crear mundo y portar una verdad, y dentro de los que se incluye, por cierto, la perspectiva, Déotte explica que el neokantismo de Cassirer y Panofsky consistió en pensar la necesidad de "puentes" culturales entre las "islas" que eran la primera y la tercera crítica kantiana, esto es, entre la estética trascendental, "que pone de manifiesto el tiempo y el espacio como condición de posibilidad de todo saber sensible puro", y la estética en el sentido de juicio del gusto. En esa misma línea, Déotte arguye que:

Es menester introducir, entre la sensibilidad pura y las síntesis espaciales a las cuales ella da lugar, y la síntesis por el entendimiento, a causa de las categorías y los conceptos, una puesta en práctica de la imaginación trascendental por los aparatos (133).

Lo que equivale a decir que es necesario introducir una puesta en práctica de una imaginación historizada. En efecto, tanto el "aparato" de Déotte como la "forma simbólica" de Cassirer introducen, a su modo, la dimensión histórica de la cultura en la síntesis de la que resulta el conocimiento y la experiencia estética. Allí radica, en efecto, la distancia que Cassirer toma respecto de Kant. Dicho de manera resumida: para Cassirer ya no hay ser en sí, sino ser simbolizado, por tanto toda reflexión ontológica se vuelve una filosofía cuyo punto de partida es la cultura. Es el símbolo el que permite que nuestro conocimiento no se limite a lo dado y trascienda el mundo inmediato de las impresiones, el símbolo entendido como energía creativa del espíritu por el que los 


\section{Macarena García Moggia}

fenómenos reciben un determinado sentido compartido. El entendimiento, entonces, no realiza la síntesis de los fenómenos solo con sus categorías, sino mediante formas simbólicas cuyo contenido cultural varía a lo largo de la historia ${ }^{2}$.

En vista de lo anterior, podemos formular lo siguiente: si el espacio que construye la ventana-perspectiva deviene forma simbólica debido a la imposición que supone de una idea del espacio por sobre la experiencia sensible del mismo, idea que es producto de unas fórmulas matemáticas que operan con un ojo abstracto y no fisiológico y que determinan, sin embargo, la percepción del mismo ¿Por qué no así el tiempo? ¿Puede hablarse, dicho de otro modo, de un tiempo simbólico correlativo a la invención de la perspectiva y, de manera más específica, ligado a la ventana-perspectiva en donde - citando una vez más las palabras de Alberti- vemos aquello que se quiere pintar, a saber, la historia? ¿Qué clase de experiencia construida del tiempo supone esa palabra, historia, como la emplea Alberti? Asimismo, ¿qué elementos nos permiten esbozar, en principio, su carácter simbólico?

II.

Hay dos versiones del texto De pictura, de Leon Battista Alberti. La primera, escrita en latín, data de 1435. La segunda, traducida por él mismo al italiano, ve la luz un año después. Entre ambas hay una diferencia importante relativa al problema que intentamos despejar. Y es que allí donde dice en el acápite número 19 de la versión en latín: Principio in superficie pingenda quam amplum libeat quadrangulum rectorum angulorum inscribo, quod quidem mihi pro aperta finestra est ex qua historia contueatur, dirá en italiano: Principio, dove io debbo dipingere scrivo uno quadrangolo di retti angoli quanto grande io voglio, el quale reputo essere una finestra aperta per donde io miri quello che quivi sarà dipinto ${ }^{3}$. Lo que cambia, lo que primero aparece $\mathrm{y}$ después no, es la palabra "istoria". Donde primero dice: "una ventana abierta a través de la cual veo la istoria", dirá después, en la versión italiana: "una ventana abierta a través de la cual veo lo que quiero pintar". La diferencia entre ambas traducciones no amerita, sin embargo, mayor detenimiento, porque, avanzado el texto, queda claro que aquello que "se desea pintar", vale decir, lo que se ve por la ventana-perspectiva no puede ser, según Alberti, sino la istoria: "La mayor obra del pintor no es un coloso, sino una istoria. La istoria le da un renombre mayor al intelecto que cualquier coloso" (Alberti, Tratado, 92).

El término comienza a introducirse en la parte media del libro segundo, ocupando un lugar prominente en el desarrollo total de la obra, lo que muestra que Alberti le daba la mayor importancia. La pintura, escribe, se debe ante todo a la istoria representada, la que resulta de un trabajo compositivo y a la que puede atribuirse por completo el efecto alcanzado en el espectador:

\footnotetext{
${ }^{2}$ Para precisar este punto, me he servido del artículo de Antonio Gutiérrez Pozo (2008).

${ }^{3}$ Las versiones originales de ambos textos fueron descargadas de la página $h t t p: / / w w w . o u s i a . i t / F e c h a ~ d e$ consulta: 4/4/ 2015 .
} 
Los cuerpos forman parte de la istoria, los miembros forman parte de los cuerpos, y los planos son parte de los miembros. Por tanto, las partes principales de la pintura son los planos. Esa gracia de los cuerpos a la que llamamos belleza, nace de la composición de los planos (...) en esta composición de planos debiera buscarse con tenacidad la gracia y la belleza de las cosas (Alberti, Tratado, 92).

Y continúa más adelante:

La fama del pintor y de su arte se halla en lo siguiente: la composición de los cuerpos. Algunas cosas que se dijeron respecto a la composición de los miembros también se aplican aquí. Los cuerpos deben armonizar entre sí en la istoria, tanto en dimensiones como en función. (...) todos los cuerpos deben corresponder en tamaño y en función a lo que está sucediendo en la istoria (97).

Cuerpos, miembros y planos organizan una composición que debe ser acorde a la istoria que se desea pintar, una istoria cuyo contenido emocional va a expresarse mediante la utilización específica de un gesto que produce identificación en el espectador. Al respecto, Alberti define una posición fundamental en la historia del arte:

La istoria moverá el alma del observador cuando cada persona representada allí muestre claramente los motivos de su propia alma. Sucede en la naturaleza que sólo ella es capaz de ciertas cosas como ella misma; derramamos lágrimas cuando lloramos, reímos con la risa y nos afligimos con las penas (100).

John Spencer, traductor de Della pintura al inglés y uno de los primeros en identificar el aspecto altamente productivo de los trabajos de Alberti, advierte que la istoria a la que se refiere el tratado estaría dirigida sobre todo hacia la expresión de un nuevo arte humanista, según esta, la adecuación de la obra a las leyes de la construcción en perspectiva da como resultado una unidad visual, temporal y espacial que asegura tanto al artista como al espectador una recompensa afectiva, de carácter moral. A este argumento, Anthony Grafton agrega que Alberti estaría brindando a la pintura humanista un valor y una función comparables a la que ostentaba, por entonces, la retórica, otro de los centros medulares del currículum humanista (37-68). Como hace notar en un exhaustivo recorrido por los elementos contextuales que permiten comprender el sentido que Alberti otorga al término istoria, el florentino volvería esto claro cuando en su escrito De re aedificatoria utiliza el término istoria para establecer una equivalencia entre la literatura y la pintura:

Cuando miro una buena pintura (hacer una mala pintura es como deshonrar una pared) experimento el mismo placer que siento cuando leo una buena historia. Ambas son trabajo de la pintura: la historia pinta con palabras, la pintura cuenta la historia con su brocha (Alberti, citado en Grafton, 42).

La comparación entre el cuadro y el libro, y más aún, entre la ventana que es el cuadro y un libro, es decisiva. Compromete, antes que nada, el problema de la 


\section{Macarena García Moggia}

narratividad, aunque solicita al mismo tiempo una pregunta, a saber: ¿cuando Alberti habla de istoria se refiere a la representación de los hechos ocurridos de manera concreta en el pasado - es la versión clásica de la palabra, que nos remite a la figura del testigo (Agamben, 133)-, o bien alude a una forma de narración independiente de los hechos? Al respecto, Grafton suscribe que el uso de la palabra historia en Alberti no puede adherirse por entero ni a una ni a otra versión: "(Alberti) dejaría en claro a los literatos que leerían el texto latino de su obra, que el término "historia" no era solo polisémico, sino inestable" (Grafton, 64). Los humanistas, en ese sentido, seguirían a sus precedentes antiguos al momento de definir la historia como un conjunto de hechos genuinos registrados por las mejores fuentes o testigos disponibles, sin embargo insistirían, al mismo tiempo, en que el estudio de la historia podía servir, de manera sumamente efectiva, para un propósito moral: "Los relatos históricos enseñaron a sus lectores los principios de la moral y la prudencia, la conducta correcta llevada a cabo decorosamente" (Grafton, 64). Estos dos aspectos de la historia pueden coexistir solo en tensión: frente a una conducta indecorosa, el historiador viola ya sea el mandamiento de decir la verdad completa, ya sea el deber de proporcionar una guía moral confiable por medio de la forma más atractiva. En esa tensión habría que ubicar a Alberti, pues:

Cuando él insiste en que la historia pintada puede tener los mismos efectos beneficiosos en la audiencia que la historia escrita -tanto si sus contenidos provienen del reino de los mitos, de antiguos hechos o de la historia sagrada- está diciendo que únicamente el estilo y la ejecución, no la correspondencia de los contenidos con la verdad, determinan la efectividad emocional de una "historia" (Grafton, 65).

De ese modo, Grafton concluye que en el texto albertiano la palabra historia puede comprenderse como la retórica misma de la pintura, si entendemos la retórica como el arte de expresar e inspirar emoción mediante un uso adecuado de la forma. Una forma que ya no se ciñe a los elementos discursivos propios de la retórica sino a elementos pictóricos, acordes a una determinada gramática visual, geométrica y corporal (a la que nos referíamos antes, a propósito de la "composición" de cuerpos, miembros y planos ${ }^{4}$ ), que no por ello deja de reconocer un vínculo tanto con los textos en los que está escrito aquello que el pintor desea trasladar al lienzo, como con "poetas, oradores y otros igualmente bien versados en las letras", que "darán creaciones nuevas o cuando menos [alguna] ayuda para arreglar bellamente la istoria" (Alberti, Tratado de pintura, 118).

Tal vez en la vereda opuesta a la de Grafton habría que situar los planteamientos de un teórico como Gerard Wajcman, autor del que quizá sea uno de los estudios más exhaustivos realizados a la fecha acerca de la dimensión "productiva", por así decirlo, de la ventana albertiana, poniendo especial énfasis en el problema de la istoria que

\footnotetext{
${ }^{4}$ Respecto del lugar de los miembros y de los cuerpos en la composición, según Alberti; y sobre la relación de la composición con la retórica, ver Baxandall (1996).
} 
escenifica. Digamos para comenzar, que según Wajcman el desplazamiento que propone Alberti de elementos discursivos a elementos compositivos propiamente pictóricos marca una "inversión topográfica" clave en la historia del pensamiento:

Viene la sospecha de que esta invención de una topografía pictórica marca un movimiento mayor del pensamiento, cuyo resultado podría ser concebir el mundo, el mundo visible de las cosas visibles, como el lugar de la acción de los hombres, cuyo nombre es historia (Wajcman, Fenêtre, 272).

Desde la perspectiva del autor, lo que Alberti plantearía es una equivalencia entre la historia, la "acción de los hombres", el "mundo visible de las cosas visibles" y el "mundo" en general. Una equivalencia, que si bien es nueva, encontraría su fundamento más en la Poética aristotélica que en la Retórica. En primer lugar, señala Wajkman, la palabra "mythe" que emplea Aristóteles funciona las veces como "histoire" según la traducción consultada. ${ }^{5}$ En segundo lugar, ambos textos entienden el mito, o la historia, como una "representación de los personajes en acción", representación que es resultado de un proceso de "mímesis poética" vinculada en el primer caso a la palabra o a la discursividad y en el segundo, el caso de Alberti, a la mirada. Wajcman se pregunta entonces: ¿Qué es una historia a la que solo tengo acceso mediante una ventana, es decir, los ojos? Si es por una ventana que puedo acceder a la historia, reflexiona el autor, entonces la historia debe contener una consistencia visual, de modo que cierta historia no sería solo del orden del lenguaje. En efecto, cuando Alberti se refiere a la historia no habla de una historia "narrativa" propiamente tal, sino de una historia que es el objeto de la pintura, vale decir, una historia visual. Así, más allá de la naturaleza híbrida del modo en que emplea el concepto "mímesis", 6 Alberti atribuye a la narración una naturaleza pictórica desde donde sería posible desplegar tanto una teoría "pictural" de la historia como una teoría narrativa de la pintura. Wajcman concluye entonces que:

La definición albertiana del cuadro como ventana abierta a la historia enuncia una ecuación esencial, fundamental, que rige un orden, posible, de la representación: que todo lo que se puede decir se puede ver, y recíprocamente. Lo visible y lo decible se recubren aquí con precisión (Wajcman, El arte, 65).

Pero habría todavía un tercer punto de encuentro -o desencuentro- entre la Poética aristotélica y el cuadro-ventana albertiano, y este tiene relación con la palabra acción, "la acción de los hombres", que es lo que se representa en la mímesis y aquello que Alberti recomendaba diversificar al máximo posible, cuidando siempre la "composición" para no caer en confusión. De acuerdo con el teórico renacentista, un cuadro presenta mayor dignidad cuando involucra un número de "cuerpos" relevante, ganando en prestigio cada vez que esos cuerpos posan y actúan de maneras distintas

\footnotetext{
${ }^{5}$ Wajcman se refiere, en particular, a la traducción de J. Lallot y J. Dupont-Roc de la Poétique, de Aristóteles.

${ }^{6}$ Ver Rougé (1997), donde se plantea un análisis de la "composición” albertiana a partir del concepto "Mímesis II", de Paul Ricoeur.
} 
(Alberti, Tratado, 40). Con ello Alberti aplicaría la metáfora de la ventana a imágenes narrativas en las que además de espacios - un espacio proyectado, objetivado y la mismo tiempo subjetivado, como decía Panofsky- se representan acciones y movimientos que en último término nos remiten a un ámbito particular, a saber, el de la representación teatral y el espacio escenográfico. Al respecto, Hans Belting ha señalado lo siguiente:

Cuando usa el término historia, Alberti no piensa simplemente en una narración, sino en una especie de representación teatral de un tema. Esta representación es la labor más noble del pintor y no es ni mera narración épica ni mero relato histórico, sino una situación teatral como la que se da entre escenario y espectador $(207)^{7}$.

Belting tiene presente, en primer lugar, la indicación de Alberti de que es preferible trabajar en formatos muy grandes para simular el tamaño real de las figuras ${ }^{8}$-como en un teatro-, lo que contribuiría a subyugar al espectador "mostrándole hombres que parezcan vivos, que sufran y amen" (Belting, 207). Por otra parte, el autor está pensando en una figura que según Alberti debe existir en cada cuadro, una figura destinada a dirigir la atención del espectador al acontecimiento representado, invitándolo con un gesto del rostro o de la mano a contemplarlo ${ }^{9}$. Ese personaje, sugiere Belting, es eminentemente teatral, cuya función es potenciar el contrato de ficción entre el espectador y los personajes de la imagen, contrato que depende de un escenario en el que vemos pintado un tema como si fuese real y que define, ante todo, una relación espacial entre la imagen y el espectador.

El cuadro como ventana y el cuadro como escenario son dos modos de figurarse el espacio simbólico que crea la perspectiva. Cada uno tiene sus derivas, que son distintas pero no excluyentes. Belting habla de una "doble representación en la que los pintores objetivan dos veces la mirada" ". Ambas formas de representación de la mirada comparten, por ejemplo, la separación entre sujeto y objeto, instaurando al mismo tiempo una distancia necesaria tanto a la representación como a la posibilidad de movilizar las facultades miméticas de los espectadores. Pero además, ambas formas de representación de la mirada implican una forma de representación de la historia de acuerdo con un lugar limitado por el encuadre que le brinda el cuadro o la ventana, lugar a partir del cual la obra constituye una unidad orgánica, cerrada sobre sí misma,

\footnotetext{
${ }^{7}$ Según aclara el mismo Belting, esta arista del problema se encuentra desarrollada con mayor profundidad en su libro Giovanni Bellini: La Pietá (Franco Cosimo Panini, Italia, 1996), que a la fecha no hemos podido consultar.

8 "Yo prefiero que practique dibujando las cosas en grande, como si la representación y la realidad fueran iguales [a escala]. En los dibujos pequeños con facilidad se ocultan grandes defectos; en lo grande se ven fácilmente los menores defectos" (Alberti, Tratado, 123).

9 'En una istoria me gusta observar a alguien que nos advierte y destaca lo que allí está sucediendo; o señala con su mano para ver; o amenaza con cara enojada y con ojos fulminantes, para que nadie se acerque; o muestra algún peligro o algo maravilloso allá; o nos mueve a reír o a llorar junto con ellos" (Alberti, Tratado, 102).

${ }^{10}$ Allí donde Panofsky habla de un "espacio simbólico" a propósito de la invención de la perspectiva, Belting hablará de una "mirada simbólica", poniendo énfasis no en el objeto sino en el sujeto (Belting, 207).
} 
donde la coherencia se funda en las relaciones y las proporciones internas de los miembros que constituyen la historia. Como argumenta Wajkman, esa unidad del lugar de representación sería una creación del cuadro, una invención de la pintura:

La unidad del lugar, que aparece como una regla formal del teatro clásico a contar del siglo XVII, es no solo una regla inventada por la pintura, heredada de la pintura, sino una regla puramente pictural. En ese sentido, el teatro clásico es pictural. El principio de unidad del lugar es la inscripción del marco del cuadro en la escritura dramática. (...) La unidad del lugar que no está en Aristóteles, está en Alberti. Es una invención de la pintura, vale decir: una invención de la ventana (Fenêtre, 288).

Si la unidad del lugar de la representación, y, por tanto, de la historia -aunque debiéramos decir: de una historia-, es una invención de la ventana, podemos pensar, todavía con Wajcman, que la ventana deviene al mismo tiempo un principio de inteligibilidad de la historia y, por tanto, del mundo.

En esa línea, Daniel Arasse plantea en El hombre en perspectiva: los primitivos italianos que la evolución de la "pintura narrativa" se inicia paralelamente al desarrollo de la historiografia florentina contemporánea. Según sostiene, existiría un parentesco profundo en el modo en que historiadores y pintores del siglo XV concibieron la narración, parentesco que redunda en una nueva forma de representar la historia según la cual el individuo humano se vuelve actor y creador de la Historia y del sentido humano del mundo (Arasse, 258-259). De cara a esa hipótesis, Wajcman dará un paso más allá, arguyendo que pintura y escritura de la historia no son simplemente dos síntomas de un cambio cultural que opera en la sociedad renacentista; desde su punto de vista, si hay entre pintores e historiadores un "parentesco profundo", este se debe a que la escritura de la historia es modificada no paralelamente sino como efecto de la pintura. Así, "si el cuadro albertiano está hecho para abrirse a la historia, la Historia podrá entonces concebirse como un cuadro" (Wajcman, 291).

III.

Pensar la pintura, y más aún, el cuadro-ventana como paradigma de la historia, implica necesariamente pensar una nueva relación del hombre con el tiempo. Como nos enseña Agamben: "Cada concepción de la historia va siempre acompañada por una determinada experiencia del tiempo que está implícita en ella, que la condiciona y que precisamente se trata de esclarecer" (Agamben, 129).

Cuando Wajcman plantea que la historia podrá concebirse como un cuadro, o bien que el cuadro, el cuadro-ventana, es aquello que origina la posibilidad de la historia, está contemplando, por una parte, al hombre como actor de esa historia, como responsable de su acción, pero además una experiencia subjetiva del tiempo que se estructura -dice parafraseando la fórmula lacaniana- por el cuadro-ventana. Por su parte, Daniel Arasse plantea que si la perspectiva es una "forma simbólica" de la nueva relación que se instaura entre los hombres y su mundo, lo es en la medida que ella 


\section{Macarena García Moggia}

designa la imagen de un mundo que es producto de un espacio pictural construido a partir de figuras en acto que dan lugar y coherencia a un mundo "humano", que sería lo mismo, para la época, que decir histórico: una historia que construye y a la vez sujeta al hombre (Arasse, 263). Una historia que es, al mismo tiempo, la historia del hombre, que lo vuelve actor y espectador de la historia y de sí mismo a la vez. Tomando en cuenta ese argumento, Wajkman anota:

Instituyendo una mirada sobre la historia, la ventana instituye una mirada sobre el mundo del hombre, sobre el mundo puesto en escena por el hombre y sobre el mundo donde el hombre es puesto en escena (296)

Con todo, ni Wajcman ni Arasse parecen tomar en cuenta que al instituirse una mirada sobre la historia $\mathrm{y}$, con ello, sobre el hombre mismo, que no hace sino observarse en esa historia a sí mismo, lo que se instaura es una relación del hombre consigo mismo que se materializa, por un lado, en la asignación de un punto de vista ideal, creado matemáticamente y basado en un sujeto cuyo lugar es designado por el plano figurativo que observa; $y$, por otro lado, por un corte arbitrario que interrumpe la "pirámide visual", marcando el límite de aquello que se va a representar, vale decir, de aquello que se va a narrar. No olvidemos las palabras de Alberti: "Primero inscribo un rectángulo de ángulos rectos tan grande como lo desee, el que se considera que es una ventana abierta en donde veo lo que quiero pintar (la istoria)". Ese límite separa aquello que queda dentro de lo que queda afuera de la historia, interrumpiendo a su vez la representación de la sucesión temporal.

Visto de esa manera, si la ventana-perspectiva simboliza una experiencia de la temporalidad, esa temporalidad no sería sino la del instante del corte de la pirámide visual, un corte instantáneo del mundo representado que implica una experiencia del tiempo concebido como un conjunto de instantes no necesariamente sucesivos, donde cada instante es la vista que a través de la ventana tenemos de la historia y, con ello, de nosotros mismos. Dicho de otro modo: el tiempo, de acuerdo con esa matriz simbólica, se configuraría y representaría como una colección de "instantáneas" que conforman, cada una, una totalidad en sí, el instante preciso de una acción visual y expresivamente compuesta, de tal forma que concentra en una imagen estática toda la potencia retórica, narrativa, de una historia.

Muy lejos entonces del período continuo y lineal que atribuimos a la escritura, la pintura se yergue, desde entonces, como una interrupción, "la caída de la lámina de la guillotina, donde soy cegado por la elección de una vista que se impone" (Déotte, 156), como se impone "un particular contenido espiritual" "a un signo sensible concreto" que acaba identificándose íntimamente con él, para emplear una vez más las palabras de Panofsky a propósito del carácter simbólico de la perspectiva.

En esa línea, podremos inferir que allí donde Panofsky piensa la relación sujetoobjeto en concordancia con la relación interior-exterior que configura la representación simbólica que la ventana-perspectiva hace del espacio, puede pensarse la relación 
sujeto-sujeto en concordancia con la relación instante-totalidad que configuraría la manera en que se simboliza el tiempo -histórico-a través del cuadro-ventana.

\author{
Pontificia Universidad Católica de Valparaíso* \\ Instituto de Arte \\ Av. Lusitania 68, Viña del Mar (Chile) \\ macarenagarciamoggia@gmail.com
}

\title{
OBRAS CITADAS
}

Agamben, Giorgio. Infancia e historia. Buenos Aires: Adriana Hidalgo, 2011.

Alberti, León Battista. Tratado de pintura. México: Universidad Autónoma Metropolitana, 1998.

Arasse, Daniel. Les primitifs italiens. Ginebra: Famot, 1968.

Belting, Hans. Florencia y Bagdad. Madrid: Akal, 2012.

Baxandall, Michel. Giotto y los oradores: la visión de la pintura en los humanistas italianos y el descubrimiento de la composición pictórica 1350 - 1450. Madrid: La balsa de la medusa, 1996.

Cassirer, Ernst. Filosofia de las formas simbólicas I. México: Fondo de Cultura Económica, 1998.

Didi-huberman, George. Ante la imagen. Murcia: Cendeac, 2010.

Déotte, Jean-Louis. La época de los aparatos. Buenos Aires: Adriana Hidalgo, 2010.

Grafton, Anthony. "Historia and Historia: Alberti's Terminology in Context". I Tatti Studies in the Italian Renaissance. Vol. 8, 1999: 37-68. Disponible en: http://www.jstor.org/stable/4603711. Recuperado el 23 de septiembre de 2014.

Gutierrez, Antonio. "La traducción simbólica de la crítica trascendental en la filosofía de Cassirer". Revista de Filosofia de la Universidad de Costa Rica 46(119), 2008: 45-55.

Kant, Immanuel. Crítica de la razón pura. Madrid: Alfaguara, 2004.

Panofsky, Erwin. La perspectiva como forma simbólica. Barcelona: Tusquets, 2010. Rougé, Bertrand. "Les deux récits du tableau: Histoire et configuration narrative en peinture". Litterature 2 (1997): 60-20.

Schefer, Jean Louis. "Préface", en Leon Battista Alberti, De pictura (1435) / De la peinture. Paris: Ėdicions Macula, 2014.

Wajcman, Gérard. Fenêtre. Chroniques du regard et de l'intime. Francia: Verdier, 2006.

Wajcman, Gérard. "El arte, el psicoanálisis, el siglo", en Aubert, et al. Lacan el escrito, la imagen. México: Siglo Veintiuno editors, 2001: 41-73. 